\title{
Microvariations de diamètre de tige pour le pilotage de l'irrigation
}

\author{
P.G. Schoch ${ }^{1}$, J.C. L'Hôtel ${ }^{1}$, P. Dauplé ${ }^{2}$, G. Conus ${ }^{1}$ et M.J. Fabre ${ }^{2}$ \\ 1 Station de Bioclimatologie; \\ 2 Unité Régionale du Service de Recherches Intégrées sur la Production Végétale, Centre de recherches d'Avignon, INRA, BP 91 , \\ F-84140 Montfavet, France
}

(reçu le 18 -1-1988, accepté le 2 -12-1988)

Résumé - Des travaux récents, Garnier (1985) et Huguet (1985), ont montré sur arbres fruitiers qu'il est possible d'utiliser la mesure des microvariations de diamètre des tiges pour piloter leur irrigation. Le but de ce travail est de montrer sur aubergine que les microvariations de diamètre de tige varient de manière synchrone avec la résistance stomatique, la photosynthèse nette et les conditions microclimatiques de la serre. Il est ainsi confirmé que cette mesure est un bon critère qui justifie son utilisation pour piloter le déclenchement de l'irrigation. Une relation linéaire entre la croissance en diamètre de la tige et la photosynthèse de la plante est mesurée tant que le fruit n'est pas noué.

assimilation nette - résistance stomatique - aubergine

Summary - Stem diameter micro-measurement for irrigation. New methods of data acquisition and processing have renewed interest in micrometric variations of stem and fruit diameter. Garnier (1985) and Huguet (1985) have recently used these techniques to monitor the internal water status of fruit trees and consequently to control orchard irrigation. The objective of our study was to improve our understanding of the daily variations in stem diameter of eggplant by simultaneously measuring stomatal resistance, net photosynthesis and the environmental conditions in a greenhouse. It appeared that stem diameter variations are an expression of the imbalance between water supply and climatic demand. This confirmed that stem diameter variation can be used as a criterion for the irrigation timing of vegetable crops. A linear relationship was found between the daily increase in stem diameter and daily plant photosynthesis before fruit-set.

net photosynthesis - stomatal resistance - eggplant

\section{Introduction}

De nombreux travaux ont été consacrés à l'étude des variations des diamètres des organes végétaux (feuilles, tiges et fruits). Ainsi les diamètres des tiges ont été étudiés en fonction des facteurs du milieu : l'évapotranspiration maximale (Waggoner and Turner, 1971), la teneur en eau du sol (Kozlowski and Winget, 1964), la résistance stomatique et le potentiel hydrique foliaire (Pereira and Kozlowski, 1976; Garnier, 1985), la transpiration (Huguet, 1985) et même la photosynthèse nette des feuilles (Schoch et al., 1987). Moins fréquentes sont les études des liaisons entre les variations de diamètre des tiges, la résistance stomatique et la photosynthèse.
Le but de notre étude est de confirmer des résultats antérieurs (Schoch et al., 1987) montrant que les mesures de microvariations des diamètres des tiges permettent de justifier cette technique pour piloter le déclenchement de l'irrigation.

\section{Matériel et Méthode}

\section{Matériel végétal et techniques culturales}

L'expérience a porté sur la variété d'aubergine «Liu Yié Qié" d'origine chinoise (Daunay et al., 1986) sous serre de façon à maîtriser l'alimentation hydrique. Le semis a eu lieu le 19 mars 1986, les plantes ont été repiquées le 15 avril au stade cotylédonaire dans des 
pots de 1 litre, puis rempotées le 29 avril dans des pots de 8 litres de terreau du commerce.

Les pots destinés aux mesures sont disposés en ligne à une densité de 6 au $\mathrm{m}^{2}$. Ils sont encadrés par des lignes de bordure. L'arrosage goutte à goutte est apporté quotidiennement pour compenser la transpiration jusqu'au 10 juin, début de la période des mesures.

Des traitements hydriques sont alors appliqués aux plantes qui sont presque au stade du premier fruit noué : 1) lot irrigué : comme pour la période de l'élevage, l'humidité du sol est maintenue à la capacité au champ; 2) lot sec : l'arrosage est arrêté à partir du 10 juin, jour que nous désignons par $\mathrm{J}$, et remis le jour $\mathrm{J}+4$.

\section{Mesures climatiques et biologiques}

Les mesures climatiques traditionnelles sont faites à l'extérieur comme à l'intérieur des serres (Schoch et al., 1987). La résistance stomatique et la photosynthèse nette sont mesurées à l'aide du Li-Cor 6000 sur des feuilles adultes bien dégagées du sommet de la plante. Chaque mesure de résistance stomatique et de photosynthèse nette présentée correspond à la moyenne de 7 feuilles prises selon un plan d'échantillonnage qui gère les variabilités climatiques d'un tunnel plastique, c'est à dire qui prenne en compte les gradients de lumière et de température. Ces mesures sont faites à $10,12,14$ et $16 \mathrm{~h}$ locales.

De même, 7 plantes sont prélevées par traitement en début et en fin d'expérience selon le même plan d'échantillonnage pour mesurer la surface foliaire par plante entière à l'aide du planimètre Li-Cor et le poids de matière sèche par plante après passage de celle-ci à l'étuve $\left(85^{\circ} \mathrm{C}\right)$ durant $24 \mathrm{~h}$.

Les microvariations des diamètres des tiges endessous de la première fleur sont mesurées à l'aide de capteurs de déplacements linéaires (Huguet, 1985) et les mesures présentées repésentent la moyenne de deux plantes par traitement, placées au milieu du tunnel plastique.

\section{Analyse statistique des données}

Les points figuratifs des résistances stomatiques et de la photosynthèse nette sont la moyenne des 7 plantes par traitement. Compte tenu de ce faible nombre de répétitions, nous avons utilisé le test non paramétrique de Steel et Dwass (Miller, 1966) pour différencier les deux traitements hydriques. Ce test permet notamment de regrouper les mesures d'heures différentes et consécutives, afin d'augmenter le nombre d'échantillons de plantes et mettre ainsi en évidence, le cas échéant, des différences significatives entre les deux traitements. La représentation graphique signale ces différences significatives par des flèches, éventuellement reliées s'il a fallu grouper les mesures pour avoir des différences significatives entre les deux traitements.

\section{Résultats}

\section{Conditions climatiques et croissance générale des plantes}

Durant la période de mesure, le temps est relativement stable et ensoleillé. Les valeurs du rayonnement global journalier sont marquées sur la Fig. 3. Les températures extrêmes de l'air oscillent régulièrement entre 18 et $26^{\circ} \mathrm{C}$ et l'humidité relative de l'air entre 50 et $80 \%$.

Pour le lot irrigué, le nombre de feuilles vertes ne varie pas durant cette période (Tableau I). La feuille jaunie de ce lot a été remplacée par une nouvelle. Les plantes du lot sec ont eu plus de feuilles jaunies pendant la période. De ce fait, il découle que la surface foliaire de chaque lot à la fin de l'essai est inférieure à celle du début avec cependant une chute plus forte pour le lot sec. L'augmentation de poids moyen de matière sèche n'est pas statistiquement différente au cours de l'essai.

\section{Résistance stomatique des feuilles (Fig. 1)}

Tout au long de l'essai, la résistance stomatique du lot irrigué ne dépasse guère $1 \mathrm{~s} \cdot \mathrm{cm}^{-1}$ entre 10 et $16 \mathrm{~h}$. Dès le jour $\mathrm{J}+2$, la résistance stomatique du lot sec augmente jusqu'à des valeurs de l'ordre de $5 \mathrm{~s} \cdot \mathrm{cm}^{-1}$ le jour $\mathrm{J}+4$, et retrouve les valeurs du lot irrigué le jour $\mathrm{J}+6$, après deux arrosages le soir de $\mathrm{J}+4$ et de $\mathrm{J}+5$.

\section{Photosynthèse nette des feuilles (Fig. 2)}

La photosynthèse nette mesurée sur les feuilles des deux traitements est identique les deux premiers jours et ne devient différente que le jour $\mathrm{J}+3$; cette différence s'accentue encore le jour

Tableau I. Croissance générale des aubergines durant la période de l'essai.

\begin{tabular}{lccr} 
Mesures & $\mathcal{J}-1$ au soir & \multicolumn{1}{l}{$J+4$ au soir } \\
\cline { 3 - 3 } & & \multicolumn{1}{c}{ irrigué } & \multicolumn{1}{c}{ sec } \\
\hline Nombre de feuilles vertes & $11,3 \pm 0,7$ & $10,0 \pm 0,8$ & $7,4 \pm 0,7$ \\
Nombre de feuilles jaunies & 0 & $1,0 \pm 0,3$ & $2,6 \pm 0,3$ \\
Surface foliaire $\left(\mathrm{dm}^{2}\right)$ & $28,7 \pm 1,7$ & $25,3 \pm 1,5$ & $17,6 \pm 1,8$ \\
Poids de matière & $39,3 \pm 2,3$ & $44,6 \pm 3,6$ & $43,1 \pm 2,1$ \\
sèche (g/plante) & & &
\end{tabular}




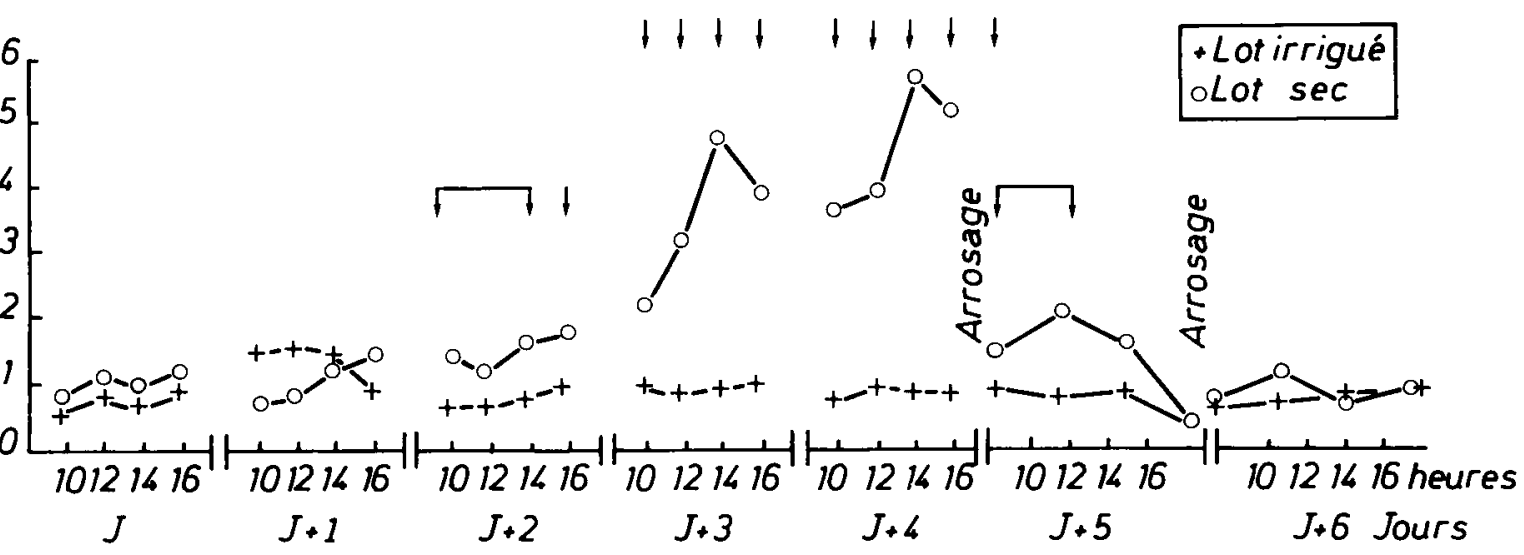

Fig. 1. Evolution de la résistance stomatique d'une feuille d'aubergine des deux lots irrigué et sec du jour $\mathrm{J}$ à $\mathrm{J}+6$. Les flèches simples ou reliées indiquent les heures où les traitements sont statistiquement différents.

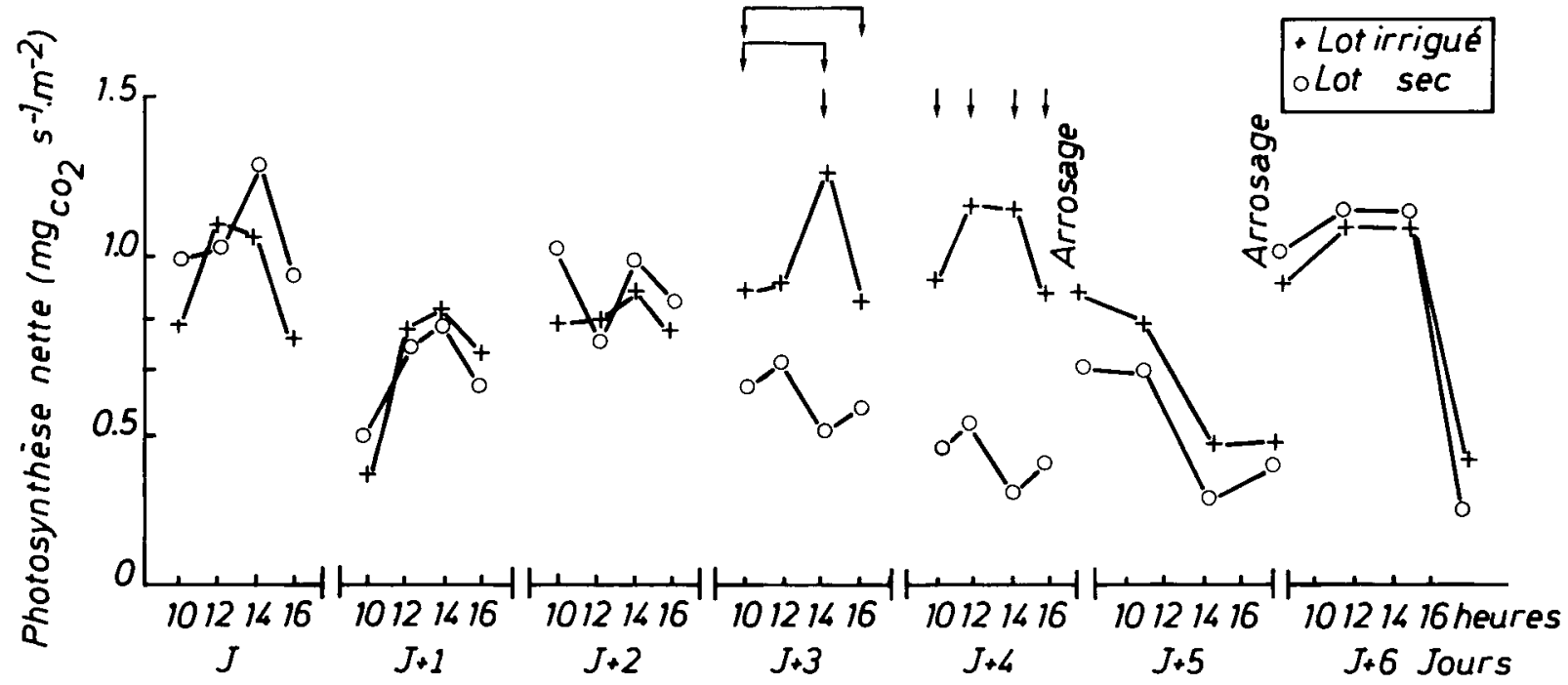

Fig. 2. Evolution de la photosynthèse nette d'une feuille d'aubergine des deux lots irrigué et sec du jour $\mathrm{J}$ à $\mathrm{J}+6$ (même convention qu'en Fig. 1).

$\mathrm{J}+4$. Après l'arrosage le soir de $\mathrm{J}+4$, la photosynthèse du lot sec le lendemain n'est pas statistiquement différente de celle du lot irrigué.

Microvariations de diamètre de la tige (Fig. 3)

Les microvariations de diamètre de la tige sont uniquement représentées durant la journée, elles traduisent les contractions journalières et les éventuelles reprises en fin de journée; durant la nuit, le diamètre augmente régulièrement pour trouver la valeur marquée au début de la journée suivante.

Pour le lot irrigué, l'amplitude des contractions journalières se situe toujours à des valeurs inférieures à $60 \mu \mathrm{m}$ et la courbe de croissance (valeurs à $8 \mathrm{~h}$ ) est toujours positive. La courbe de variation de diamètre du lot $\mathrm{sec}$ suit approximativement celle du lot irrigué durant les trois premiers jours. A partir du jour $J+3$, les contractions $\mathrm{du}$ lot sec augmentent, de $170 \mu \mathrm{m}$ pour le jour $\mathrm{J}+3$ et de plus de $500 \mu \mathrm{m}$ pour $\mathrm{J}+4$. Egalement à partir de $\mathrm{J}+3$ au matin, on constate une diminution du diamètre par rapport à la veille à la même heure et ce phénomène se poursuit jusqu'à l'arrosage. Dès l'arrosage du lot sec, le diamètre récupère essentiellement la nuit mais retrouve le jour $\mathrm{J}+6$ une valeur inférieure à celle du lot irrigué, avec cependant une contraction journalière à peu près équivalente au témoin.

\section{Discussion et Conclusion}

Après une discussion sur la validité des différentes mesures nous comparerons ces résultats entre eux.

\section{Croissance générale et photosynthèse}

Le nombre de feuilles par plante n'a statistiquement pas varié pour les deux lots au cours de l'essai. Cependant pour le lot sec, certaines feuilles anciennes ont jauni et la surface foliaire 


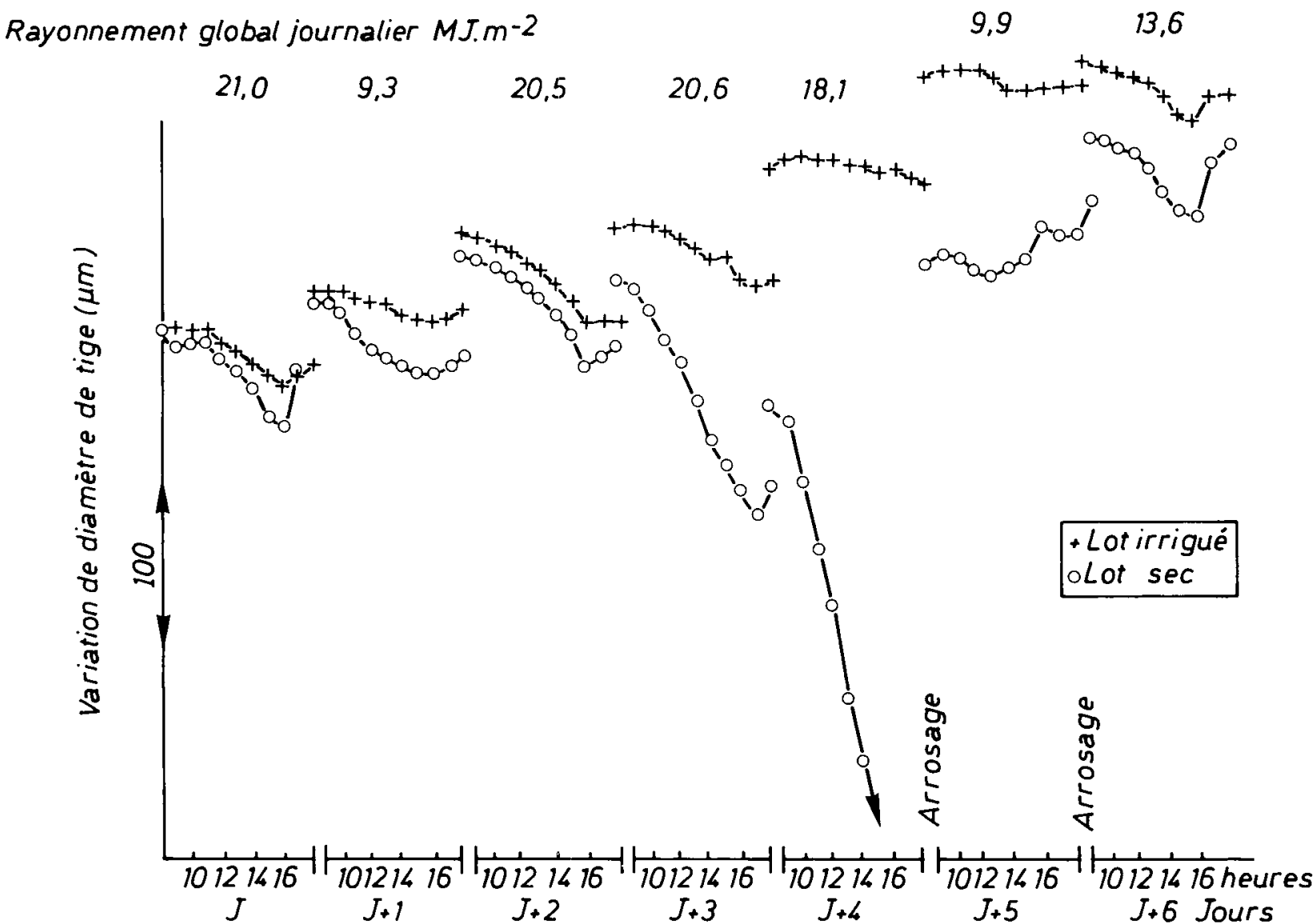

Fig. 3. Rayonnement global journalier et variations de diamètre de la tige d'aubergine des deux lots irrigué et sec.

de ces plantes a été réduite de ce fait. Le poids de matière sèche d'une plante a augmenté pour les deux lots, mais cette augmentation n'est pas statistiquement significative. Néanmoins pour le lot irrigué, cette différence entre $\mathrm{J}+1$ au soir et $\mathrm{J}+3$ au soir est en moyenne de 5,3 g/plante.

En prenant comme hypothèse tout à fait simpliste que la photosynthèse nette d'une plante est la photosynthèse nette de la feuille mesurée multipliée par la surface foliaire de la plante, il est possible de calculer la photosynthèse nette cumulée durant le temps de l'expérience, soit $30,9 \mathrm{~g} /$ plante. En admettant également la relation simpliste que la respiration nocturne représente la moitié de la photosynthèse de la veille, il reste encore environ $15 \mathrm{~g} /$ plante à répartir entre la partie aérienne et les racines. Si nous admettons également que cette répartition se fasse par moitié, l'augmentation de poids de matière sèche durant cette période doit donc être d'environ 7,5 g/plante.

Compte tenu de toutes les approximations admises, nous pouvons considérer que ces 5,3 $\mathrm{g} /$ plante directement mesurés sont très voisins des $7,5 \mathrm{~g} /$ plante calculés.

\section{Succession des manifestations du manque d'eau}

Nous retrouvons le fait qu'une plante d'aubergine bien alimentée en eau ne présente pratiquement pas de régulation stomatique en milieu de journée (Daunay et al., 1986; Schoch et al., 1987). La contrainte hydrique se manifeste à partir du jour $\mathrm{J}+2$ où la résistance stomatique du lot sec est significativement supérieure à celle du lot irrigué. Très rapidement après l'arrosage, la résistance stomatique du lot sec rejoint les valeurs du témoin.

Statistiquement, la photosynthèse nette du lot sec se distingue du lot irrigué à partir du jour $\mathrm{J}+3$. Cette différence s'accentue également le jour suivant pour redevenir presque normale après l'arrosage le jour $J+5$. II est donc logique de situer le manque d'eau pour la photosynthèse le jour $\mathrm{J}+3$. 
L'observation des variations de diamètre des tiges montre clairement que, durant les trois jours jusqu'à $J+2$, il n'y a pas de différence entre les deux lots : les contractions journalières sont de même ordre de grandeur et la croissance nette d'une journée sur l'autre est à peu près la même. Le matin du jour $\mathrm{J}+3$, la croissance en diamètre du lot sec est négative, c'est-à-dire que le diamètre le jour $\mathrm{J}+3$ est inférieur à celui du jour $\mathrm{J}+2$, et au cours de cette journée, la contraction sera plus de trois fois plus importante que celle du lot irrigué. Ces différences, contraction diurne et croissance négative le lendemain, s'amplifient encore le jour $\mathrm{J}+4$; une journée après l'arrosage, la croissance du lot sec redevient semblable à celle du lot irrigué. En définitive, le diamètre du lot sec est inférieur à celui du lot irrigué.

En se basant sur les variations de diamètre, il est donc possible de repérer dans cette expérience, un manque d'eau à partir du jour $\mathrm{J}+3$, d'une part en le comparant à un lot toujours bien irrigué, d'autre part en notant le jour où la croissance en diamètre devient négative. Ce dernier critère a l'avantage de ne pas nécessiter les valeurs d'un témoin.

Rappelons qu'en se basant sur la régulation stomatique, la manifestation du manque d'eau se situe le jour $\mathrm{J}+2$, et pour la photosynthèse nette seulement le jour $\mathrm{J}+3$.

Cette succession paraît logique : un manque d'eau entraîne la régulation stomatique, puis les échanges gazeux et enfin la photosynthèse. Ce manque d'eau entraîne la mobilisation des réserves hydriques de la plante, notamment de la tige, qui voit son diamètre diminuer significativement et ce dernier marque une croissance négative apparente qui disparaît dès que l'eau est à nouveau disponible pour la plante.

La mesure de la croissance en diamètre de la tige permet de définir deux critères (absence de croissance nocturne, contraction diurne importante, qui justifient son utilisation pour piloter le déclenchement de l'irrigation (Garnier, 1985; Huguet, 1985; Schoch et al., 1987). En effet, dans cette expérience, ces critères agissent en synchronisme avec les autres paramètres physiologiques classiques qui sont bien plus difficiles à mettre en cuvre. De plus, sans témoin, il est possible de conclure à un manque d'eau lorsque la croissance de nuit ne se fait plus après une journée à forte contraction.

Dans cette expérience, il est possible de se baser sur l'un ou l'autre des deux critères (absence de croissance nocturne, contraction diurne importante) pour déclencher l'irrigation. II n'en serait plus de même en cas de cuiture hydroponique à arrosages journaliers intermittents. II fau- drait alors se baser uniquement sur les contractions diurnes et définir expérimentalement la valeur maximale à ne pas dépasser.

\section{Diamètre de la tige et photosynthèse nette}

En reprenant le modèle simpliste de la photosynthèse d'une plante à partir de la photosynthèse d'une feuille, nous obtenons (Fig. 4) pour le lot irrigué une très bonne corrélation linéaire entre le diamètre de la tige (valeurs en début de journée) et la photosynthèse nette cumulée les jours précédents, du jour $\mathrm{J}$ à $\mathrm{J}+6$ (coefficient de corrélation 0,94 ). Pour les jours suivants, la courbe s'infléchit et cela est vraisemblablement dû à la présence du fruit qui devient un organe de remplissage préférentiel des métabolites de la photosynthèse. Le passage rapide d'une relation linéaire à une totale indépendance mérite confirmation.

Cette relation linéaire entre le diamètre de la tige et la photosynthèse nette cumulée durant le stade végétatif traduirait le fait qu'au cours d'une journée à faible rayonnement, le diamètre augmenterait peu, contrairement à l'élongation des entre-nœuds qui est généralement stimulée par une faible lumière (Lecharny et Jacques, 1974). La tige s'allonge par manque de lumière sans modification de son diamètre. Si ces résultats devaient se confirmer, la mesure de la croissance en diamètre de la tige, durant le stade végétatif, pourrait donc devenir un critère d'appréciation de la photosynthèse du plant de tomate et peutêtre permettre de piloter les conditions microclimatiques, notamment thermique d'une serre.

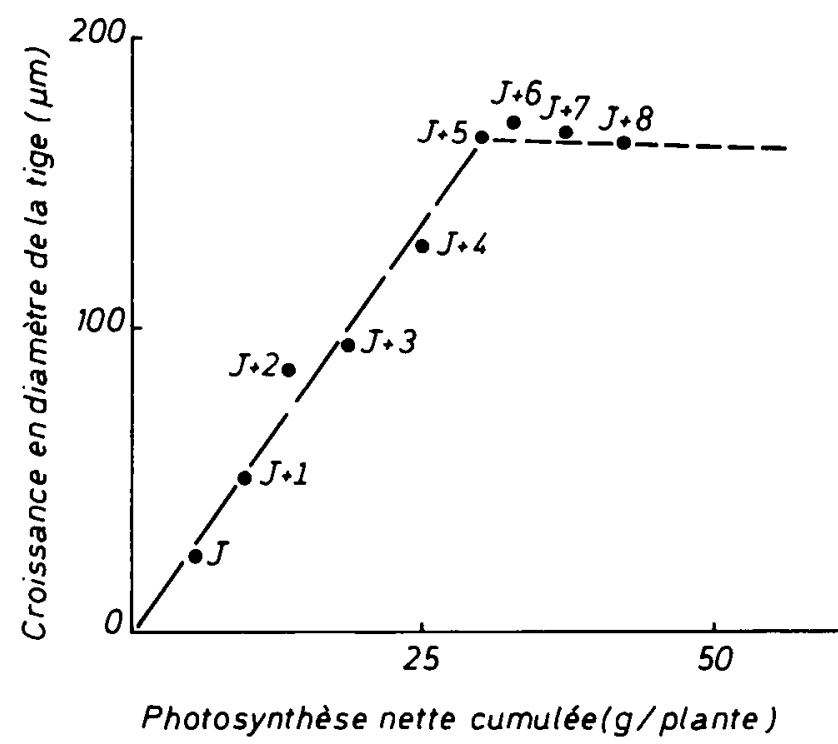

Fig. 4. Croissance en diamètre de la tige d'aubergine du lot irrigué en fonction de la photosynthèse nette cumulée du jour $\mathrm{J}$ à $\mathrm{J}+8$. 


\section{Remerciements}

Ce travail a fait l'objet d'un soutien financier de l'ONIFLHOR. Qu'il en soit sincèrement remercié. Cet article a été présenté au Colloque franco-israélien du 19 au 23 octobre 1987 à Bordeaux.

\section{Références}

Daunay M.C., Malet P. \& Schoch P.G. (1986) Facteurs agroclimatiques influençant la résistance stomatique de l'aubergine (Solanum melongena L.). Agronomie, 6, 615-622

Garnier E. (1985) Dessèchement du sol et indicateurs physiologiques de l'état hydrique chez le pêcher : application au déclenchement de l'irrigation. Thèse de Docteur-Ingénieur en Agronomie, ENSA de Montpellier.

Huguet J.G. (1985) Appréciation de l'état hydrique d'une plante à partir des variations micrométriques de la dimension des fruits ou des tiges en cours de journée. Agronomie 5, 733-741
Kozlowski T.T. \& Winguet C.H., (1964) Diurnal and seasonal variation in radii of tree stems. Ecology 45 , 149-155

Lecharny A. \& Jacques R. (1974) Phytochrone et croissance des tiges; variations de l'effet de la lumière en fonction du temps et du lieu de photoperception. Physiol. Vég. 12, 721-738

Miller R.G. (1966) In: Simultaneous statistical inference. Mc Grow-Hill, New-York

Pereira J.S. \& Kozlowski T.T., (1976) Diurnal and seasonal changes in water balance of Abies balsamea and Pinus resinosa. Oecol. Plant. 11, 397-412

Schoch P.G., Katerji N, Rimgoto P., Tchamitchian M., Malet P., L'Hôtel J.C. \& Daunay M.C. (1987) Influence du niveau d'alimentation hydrique sur les variations du diamètre des tiges, du potentiel hydrique, de la résistance stomatique, de la transpiration et de la photosynthèse de l'Aubergine (Solanum melongena L.). Agric. For. Meteorol. 40, 89-104

Waggoner P.E. \& Turner N.C. (1971) Transpiration and its control by stomata in a pine forest. Conn. Agric. Exp. Annu. Bull. 726, 26-34 